\title{
The interface between general and forensic psychiatry: the present day ${ }^{\dagger}$
}

\author{
Vivek Khosla, Phil Davison, Harvey Gordon \& Verghese Joseph
}

\begin{abstract}
SUMMARY
With the subspecialisation of psychiatry in the UK, clinicians encounter problems at the interfaces between specialties. These can lead to tension between clinicians, which can be unhelpful to the clinical care of the patient. This article focuses on the interface between general and forensic psychiatry in England and Wales. The pattern of mental health services in England and Wales differs to an extent from those in Scotland, Northern Ireland and in the Republic of Ireland. Consequently, the interface between general and forensic psychiatry is subject to varying influences. Important interface issues include: the definition of a 'forensic patient'; the remit and organisation of services; resources; clinical responsibility; and care pathways. This article also discusses a general overview of how to improve collaboration between forensic and general adult psychiatric services.
\end{abstract}

\section{LEARNING OBJECTIVES}

- Develop an understanding of important issues at the forensic/general adult psychiatry interface.

- Be aware of areas of conflict that may arise at the forensic/general adult psychiatry interface.

- Be aware of options for optimum cooperation at the interface.

\section{DECLARATION OF INTEREST}

None.

In our previous article (Gordon 2014, this issue) we discussed the evolution of forensic psychiatry in the UK up to the 1970s. Here we focus on more recent history and the present day collaboration and tension between the two.

The Mental Health Act 1959 made it legal for the then 'special hospitals' (Broadmoor, Rampton and Moss Side) to accept civilly detained patients who posed a risk of serious harm to others. 'Forensic' patients thereafter were not limited to people with mental illness who had been charged with or had carried out criminal offences (mentally disordered offenders). An individual could become a forensic patient on the basis of their perceived level of potential dangerousness to others. Risk to others became the main parameter guiding whether a psychiatric patient was cared for by general or forensic services. A related but separate enduring issue is which people even qualify as psychiatric patients at all. This especially applies to people with personality disorder who show elements of antisocial behaviour and those with paraphilias.

Since their advent in the mid-1970s, medium secure units have become the main forensic interface with general psychiatry. A new forensic intraface has arisen between medium secure units and high secure hospitals where tension and collaboration are similarly evident. Such a dynamic cannot be eliminated, it can only be managed according to how psychiatric services are configured.

\section{The general/forensic interface}

The seminal paper of relevance is that of John Gunn (1977). Its timing was not coincidental. The 1970s had seen several key factors: homicides committed by a man a year after he was discharged from Broadmoor (Bowden 1996); increased resistance by general psychiatric hospitals to accepting patients on transfer from special hospitals (Dell 1980), which consequently became overcrowded; and the beginning of the programme of regional medium secure provision that would take a generation to reach adequate levels.

Gunn described two models of care for mentally disordered offenders: the parallel model and the integrated model. In the parallel model, patients discharged from forensic units to the community would remain the responsibility of forensic clinicians. In the integrated approach, such patients would be transferred back to general adult services. There were also individuals released from prison into the community who had mental disorders and were in need of care and supervision, but who had never been in a psychiatric hospital. In an entirely parallel system, no patients discharged from a forensic unit would ever be transferred back to general psychiatric services. As a result,

\section{ARTICLE}

Vivek Khosla is a consultant forensic psychiatrist at Littlemore Mental Health Centre, Oxford Health NHS Foundation Trust. He has an interest in optimising the interface between general and forensic psychiatry. Phil Davison is a consultant psychiatrist employed by the Oxford Health NHS Foundation Trust. He has worked as a consultant general adult psychiatrist for 17 years, and throughout this time, he has valued his working relationship with his forensic psychiatry colleagues. Harvey Gordon, now retired, has worked as a consultant forensic psychiatrist at Broadmoor Hospital the Maudsley and Bethlem Royal Hospitals, and Oxford's Littlemore Mental Health Centre. He has an interest in the history of psychiatry and forensic psychiatry. Verghese Joseph is a consultant psychiatrist at Stamford Resource Centre,

Stamford, and the clinical lead for research at Lincolnshire Partnership NHS Foundation Trust. His research interests include understanding abnormalities in brain function in schizophrenia using functional magnetic resonance imaging and evoked response potentials. Correspondence Dr Vivek Khosla, Oxford Clinic, Oxford Health NHS Foundation Trust, Littlemore Mental Health Centre, Oxford OX4 4XN, UK. Email: vivek.khosla@oxfordhealth. nhs.uk

${ }^{\dagger}$ See pp. 350-358, this issue 
the forensic service would increasingly expand and become unmanageable. The very existence, however, of a separate forensic service would be likely to generate banishment pressure from general adult services, including reluctance to take back patients even when their level of risk had declined. And if risk were the main factor determining placement, some patients pose a higher risk in the shorter term, whereas others are generally quiescent but then relapse into serious violence after a considerable time. There was also greater stigma attached to the forensic patient that arose from both their psychiatric history and their criminality or antisocial behaviour, rendering such patients unpopular with general services.

\section{The 1970s and 1980s}

The first two decades of the provision of medium secure services did not resolve all of the problems at the interface with general adult psychiatry. The early pioneers in medium secure units felt that many referrals from general psychiatric units were essentially of civil patients with schizophrenia who were difficult to manage but not 'forensic'. Some had had their diagnoses changed from schizophrenia to personality disorder, which would render them, at that time, unsuitable for general psychiatric services (Higgins 1991). However, if the

B0X 1 Main publications on the modern general adult/forensic interface

\begin{tabular}{ll}
\hline Home Office \& Department of Health and & McClelland et al (2001) \\
Social Security (1975) & Buchanan (2002) \\
Gunn (1977) & Burns (2002) \\
Higgins (1981) & Holloway (2002) \\
Snowden (1985) & Snowden (2002) \\
Scannell (1989) & Szmukler (2002) \\
Gallwey (1990) & Tighe et al (2002) \\
Higgins (1991) & Judge et al (2004) \\
Higgo \& Shetty (1991) & Mohan et al (2004) \\
Department of Health \& Home Office (1992) & Dowsett (2005) \\
Murray (1996) & Turner \& Salter (2005) \\
Whittle \& Scally (1998) & Mohan \& Fahy (2006) \\
Birmingham (1999) & Ozdural (2006) \\
Harding \& Cameron (1999) & Coid et al (2007) \\
Snowden et al (1999) & Malik et al (2007) \\
Chaloner \& Coffey (2000) & O'Grady (2008) \\
Vaughan et al (2000) & Turner \& Salter (2008) \\
Buchanan (2001) & Bradley (2009) \\
Burns (2001) & Humber et al (2011) \\
Bowden (2001) & Natarajan et al (2012)
\end{tabular}

new medium secure units were to be successfully responsive to the criminal justice system, they could not become a long-term secure intensive care system for the National Health Service (NHS). At the other end of the security spectrum, the policies of medium secure units to seek to limit admissions to no more than 2 years and the disinclination to accept patients with personality disorder rendered their relationship with special hospitals only partially complementary.

\section{The 1990s}

By the 1990s, it was evident that high and medium secure services in forensic psychiatry needed to be complemented by services offering a lower level of security to safely manage and treat many patients (Cripps 1995; Reed 1997). Low secure provision was similarly required to support general adult psychiatric patients who show disturbed behaviour and/or frequent absconding. The management of low secure units would be undertaken by either general or forensic services or potentially shared by both (Turner 2008).

The 1990s also saw the appearance in the UK of psychiatric intensive care units (PICUs). These are essentially a form of secure provision intended for short-term containment or treatment of psychiatric patients manifesting disturbed behaviour. General or forensic patients needing longer periods of detention in locked conditions have clinical needs that may compromise the functioning of PICUs (Perreira 2001).

\section{Issues arising from the modern general adult/forensic interface}

The literature (Box 1) tends to focus on the following questions:

- What does the term 'forensic patient' mean?

- Are the procedures for regulating the transfer of patients between general and forensic services appropriate?

- On discharge from a secure facility, should the patient be cared for by forensic or general psychiatric services?

- Is the emphasis on risk assessment a distraction from the objective of providing optimal care for all psychiatric patients?

- Is there any need for a separate subspecialty of forensic psychiatry?

\section{What does the term 'forensic patient' mean?}

Subspecialisation of psychiatry in the UK is based on age (e.g. child and adolescent or old age psychiatry), or a specific clinical diagnosis (e.g. intellectual disability, addiction) or a 
specific treatment modality (e.g. psychotherapy). However, the clinical domains and patient age groups of forensic and general psychiatry overlap and it is therefore not easy to draw boundaries between these two specialties (Szmukler 2002). The definition of a forensic patient is no easier to formulate than that of insanity (historically) or of mental illness or mental disorder. Neither is it any easier to define what a general psychiatric patient is. When the Reed Committee reported in the early 1990s, it referred to 'mentally disordered offenders and those requiring similar services' (Department of Health 1992). However, 'mentally disordered offenders' includes not only those convicted but also those awaiting trial. Those 'requiring similar services' refers to patients not within the criminal justice system but who pose a risk to others as a result of, or along with, their mental disorder.

The distinction between mental illness and mental disorder also has some bearing on the interface between general and forensic psychiatry. Psychiatrists vary in their confidence in the efficacy of the treatment of patients with personality disorders. Even within forensic psychiatry, considerable ambivalence exists regarding their treatability, although not infrequently there is comorbidity with elements of affective disorder, psychosis, eating disorders and neurological impairment (Dolan 1993; National Collaborating Centre for Mental Health 2010). The treatment of paraphilias, ${ }^{\ddagger}$ whether by general or forensic psychiatrists, is also frequently incomplete, without full appreciation of the need for biological methods (World Federation of Societies of Biological Psychiatry 2010).

\section{The risk definition}

A working definition of a forensic patient may therefore be any patient with a mental disorder who poses a serious risk to others or who is simply considered unsuitable by general psychiatry but accepted by forensic services. What level of risk makes a patient 'forensic' is in itself a matter of judgement for the individual clinician and the service. In clinical practice, mentally disordered offenders who have committed serious violent or sexual offences come under the remit of forensic services. These patients often present with low frequency but high impact risk to others, the risk extends well into the future, and they have complex, long-term, multidisciplinary treatment needs. Disagreements may arise between forensic and general services when patients present with high and frequent risk of less severe violent offending. Such patients are not always charged with criminal offences. Where both general
BOX 2 Factors that favour care of a mentally disordered patient by forensic services

- History and high risk of serious interpersonal violent and/or sexual offence

- Extensive history of criminality and antisocial behaviour

- Significant personality factors and substance misuse relevant to risk, in addition to the mental illness

- Need for complex, long-term, multidisciplinary treatment including offence-related work

- Patients managed under sections 37, 41 or other sections of the Mental Health Act 1983 (as amended in 2007) with Ministry of Justice restrictions

- Media interest in the individual patient

and forensic services do not provide treatment, historically it is the prison service that has ended up meeting the task. Factors that favour care of a mentally disordered patient by the forensic service are listed in Box 2.

\section{Are the procedures for regulating the transfer of patients between general and forensic services appropriate?}

Nowadays, the commissioning arrangements for forensic and general services are different. The case-loads of general adult psychiatrists are considerably higher than those of their forensic psychiatric colleagues. For a small minority of patients on general psychiatrists' case-loads, where there is concern about risk, a referral may be made for a risk assessment or for transfer to secure services. The gatekeeper for such a transfer is usually the clinical director of forensic services or, where there is no separate forensic directorate, it may be the lead forensic clinician.

In a paper of 2008, Turner $\&$ Salter asked why the right to admit to medium secure units should be exclusively confined to forensic specialists. They make a persuasive point. If a general adult psychiatrist feels that one of their patients cannot be safely cared for in a general psychiatric unit or is unsuitable for a PICU, there is merit in their having access to admission to a medium secure unit. Once admitted, the patient would come under the care of one of the forensic psychiatrists. During the patient's stay in medium security, the general adult psychiatrist and team would continue to maintain contact with the patient and attend clinical reviews. After sufficient stabilisation, collaboration would be required to determine whether the patient can be transferred back to general psychiatry or cared for by the community forensic team.
FFor a recent discussion of paraphilias in Advances, see Yakeley J, Wood H (2014) Paraphilias and paraphilic disorders: diagnosis, assessment and management, 20 202-213. Ed. 
An alternative is that forensic psychiatry retains sole admission rights to medium security (and low security if within the forensic directorate), but that if a general psychiatric patient is declined such a transfer, an appeal mechanism is available that can override the original decision. Psychiatrists should not feel reticent about making such appeals, as a properly conducted review may result in improvement in the system. The patient should also have access to the appeal process. Although managers' hearings and mental health tribunals comment frequently on placement, there is additional merit in clinicians and patients having an appeal mechanism.

\section{Community patients}

The previous section relates to in-patients, but similar issues are pertinent to patients in the community, for whom a range of models exist throughout the country. In all cases, a referral to the forensic service for advice on risk and management results in an assessment and a detailed report by the forensic service. This can take several weeks. Although such detailed assessment is appropriate in many cases, it may not be required for each and every case. Regular liaison meetings between general and forensic colleagues to discuss individual cases can reduce the need for full assessments in some cases. They can be valuable for joint working and making decisions about access to services and also help in the management of difficult patients under the care of general colleagues.

\section{On discharge from a secure facility, should the patient be cared for by forensic or general psychiatric services?}

A key issue in this debate is that of current risk weighed against historical risk. Some mentally disordered offenders who have committed homicide during a psychotic state, especially where there was little or no prior criminal history, pose little or no further risk as long as they do not relapse. This may depend on adherence to medication, including its mode of administration, and, where appropriate, abstinence from substance use. Some homicides by patients discharged from psychiatric hospitals have followed either changes in diagnosis from psychosis to personality disorder and a consequent stoppage of prescriptions of medication (Shetty 2012), or changes from depot to oral medication authorised by the psychiatrist but then not taken by the patient (Scotland 1998). For patients with high historic risk, it seems advisable for discharge into the community to be supervised by the forensic community team for at least 2 years before any consideration of transfer back to general services.

The care of patients discharged from secure services into the community with diagnoses of personality disorder and/or paraphilias is more complex. The rate of recidivism for patients categorised previously as having psychopathic disorder is higher than that for patients with mental illnesses. Multidisciplinary and multiagency involvement in multi-agency public protection arrangements (MAPPA) meetings can be of considerable value. A lengthy period of stabilisation in the community managed by forensic services is clearly preferable for these patients. The risk level in some of these patients only subsides with increasing age and they require careful supervision.

\section{Paraphilias}

Although patients with paraphilias have in the past been treated by some general adult psychiatrists, those who have committed serious sexual offences may well be more appropriately treated by forensic services. Dynamic psychotherapy may be helpful, but the current evidence points to a combination of cognitive-behavioural therapy and anti-libido medication having the optimal effect (Gordon 2004). Such patients could be safely transferred from forensic to adult general community teams after some years of stabilisation in the community and subject to the ongoing monitoring of the paraphilia and maintenance of appropriate treatment.

Is the emphasis on risk assessment a distraction from the objective of providing optimal care for all psychiatric patients?

A number of general psychiatrists have noted that a disproportionate amount of the mental health budget is spent on a minority of psychiatric patients who pose a high level of risk to others (Goldberg 2006; Holloway 2011). There is an additional issue of whether community forensic teams are any more effective than general adult community mental health teams in caring for patients discharged from secure services (Coid 2007; Humber 2011). Some general adult psychiatrists regard risk to others as primarily a responsibility for agencies such as the police, with the doctor's role focusing solely on the therapeutic care rather than control. In practice, it is better to look upon both as having as role in maintaining safety.

That forensic patients cost more to contain and treat is not a new phenomenon (Cross 1876). Only 13 years after the opening of Broadmoor there were complaints from local asylums that 
more was spent per patient in the criminal lunatic asylum than in local asylums. A government commission, however, regarded the extra cost as necessary (Criminal Lunacy (Departmental) Commission 1882). It is probably inevitable that the nature and severity of some mental or physical disorders render them more expensive to treat than others, especially where higher numbers of staff are required.

A more pertinent issue is whether community forensic teams are any more effective than general adult community mental health teams. The study by Coid et al (2007) found no advantages in terms of rates of readmission or of reconviction. Humber et al (2011) found that those on the case-loads of community forensic teams had higher levels of historical risk but similar levels of current risk. They suggested transfer of such patients back to general adult community teams, leaving only those presenting with the highest levels of current risk with forensic community teams. A doubleblind trial of parallel versus integrated community care was proposed, but its ethical feasibility might be problematic.

Many psychiatrists are clearly frustrated by the safety culture that British society has adopted over the past 20 years. However, psychiatry must be responsive to the social context. The Hippocratic dictum to first do no harm can arguably be said to apply not only to the health of the individual patient but also to society as a whole. In the case of mentally disordered offenders and similar patients, the doctor's role is inevitably concerned not only with the patient's health and safety but also with that of people who may become the patient's victims.

\section{Is there any need for a separate subspecialty of forensic psychiatry?}

As discussed in more detail in our previous article (Gordon 2014, this issue), the origins of forensic psychiatry in Britain can be traced to the early 19 th century, with the development of medicolegal expertise in the courts and the opening of specialised facilities for the criminal lunatic. The provision of medical and psychiatric care in prisons was a further factor. It is notable, however, that a separate subspecialty of forensic psychiatry did not start to take form until the transformation of the Royal Medico-Psychological Association into the Royal College of Psychiatrists in the early 1970s (Bewley 2008). Psychiatrists today may be expert witnesses in court, work in prisons, or even work in forensic psychiatric facilities without necessarily being fully trained forensic psychiatrists. John Bucknill, a medical superintendant in a local asylum in the 1850 s, stated that it was not selfevident that there was any real difference between 'lunacy' and 'criminal lunacy' (Bucknill 1851). But the era of psychiatrists as generalists who could assess any patient, whether a child, adolescent or elderly person, is now behind us and specialisation and even superspecialisation, such as old age forensic psychiatry (Yorston 1999), is now the direction of configuration of psychiatric services.

\section{Some further practical aspects of managing the general adult/forensic interface}

\section{Trust protocols}

Protocols can be devised and agreed by medical advisory committees and other health professional groups within a trust regarding the placement of patients in general adult or forensic facilities. A trust's executive board can ultimately agree such protocols across the trust. These protocols can be audited and reviewed annually.

\section{Accessibility of forensic advice}

Forensic psychiatrists should be readily available on the telephone for advice on specific cases, or by invitation to general adult clinical reviews. A regular opportunity for general adult psychiatrists or members of their teams to discuss cases with the forensic team is also most useful.

\section{Adverse incident reviews}

Regular reviews within a trust can consider adverse incidents, including those at the general/ forensic interface. Such meetings can be stressful for staff involved in an adverse incident, but with sensitive management and chairing, these reviews are helpful provided that all relevant issues are discussed openly.

\section{Conclusions}

As this and our previous article (Gordon 2014, this issue) have shown, the interface between general and forensic psychiatry is not new. It has, however, evolved over time with the development of services. There are areas of tension and potential conflict at the interface, but also opportunities for collaboration for the benefit of patients. Cooperation and communication between psychiatrists are essential to the effective management of this interface between services.

\section{Acknowledgement}

We thank Dr Natasha Gopee, Consultant Community Forensic Psychiatrist, Lincolnshire Partnership NHS Foundation Trust, for helpful comments on the first draft of this article. 
Szmukler G (2002) Interface between forensic and general psychiatry in the community. Criminal Behaviour and Mental Health, 12: 73-9.

Tighe J, Henderson C, Thornicroft G (2002) Mentally disordered offenders and models of community care provision. In Care of the Mentally Disordered Offender in the Community (ed A Buchanan): 89-110. Oxford University Press.

Turner T, Salter M (2005) What is the role of a community forensic mental health team? Psychiatric Bulletin, 29: 352

Turner T, Salter M (2008) Forensic psychiatry and general psychiatry: reexamining the relationship. Psychiatric Bulletin, 32: 2-6.
Vaughan PJ, Pullen N, Kelly M (2000) Services for mentally disordered offenders in community psychiatric teams. Journal of Forensic Psychiatry, 11: $571-86$.

Whittle MC, Scally MD (1998) Model of forensic psychiatric community care. Psychiatric Bulletin, 22: 748-50.

World Federation of Societies of Biological Psychiatry (2010) Guidelines for the biological treatment of paraphilias. World Journal of Biological Psychiatry, 11: 604-55.

Yorston G (1999) Aged and dangerous: old-age forensic psychiatry. British Journal of Psychiatry, 174: 193-5.
MCOs

Select the single best option for each question stem

1 Which of the following statements is true?

a special hospitals became legally able to accept patients detained under civil sections as a result of the Mental Health Act 1959

b medium secure units have been available in the UK since the mid-1950s

c in the Reed Report (1992), the term mentally disordered offender referred to an offender with mental illness only

d in the 1980s, general psychiatric hospitals were very willing to accept patients on transfer from special hospitals

e the cost per patient per year is the same for forensic and general adult services.

2 Which of the following statements is true?

a patients with a diagnosis of antisocial personality disorder should only be admitted to a forensic service

$\mathrm{b}$ the rate of recidivism among patients categorised previously as having psychopathic disorder is higher than that among patients with mental illness c paraphilias are not mental disorders

d the optimum treatment for paraphilias is dynamic psychotherapy

e risk assessment of violent behaviour in psychiatry can only be done by a forensic psychiatrist

3 Which of the following is not likely to help resolve disagreements at the forensic/ general adult service interface?

a a protocol for management of patients across services

b no mechanism for appeal in cases of disagreement

c regular liaison between colleagues

$\mathrm{d}$ an audit of referrals to the forensic service

e serious case reviews.

4 A patient is suitable for forensic services if: a the patient is a suicide risk

b the patient has committed homicide

c the patient is not on a restriction order (section

41 of the Mental Health Act)

$\mathrm{d}$ the patient has an 10 below 70 .

e the patient has a history of substance misuse.
5 Which of the following statements is true?

a community forensic mental health teams usually have higher case-loads than general adult community mental health teams

b community forensic mental health teams have been consistently shown to be more effective in reducing reconviction rates

c community forensic mental health teams have been consistently shown to be more effective in reducing readmission

$d$ research has shown that patients under the care of community forensic teams have higher levels of historical and current risk than patients under the care of general adult community mental health teams

e research has shown that patients under the care of community forensic teams have higher levels of historical risk and similar levels of current risk compared with patients under the care of general adult community mental health teams.

\section{Correction}

Russ T (2014) Hangover Square by Patrick Hamilton. Advances in Psychiatric Treatment, 20: 247-9.

On p. 248, the first quotation in the second column should read:

'Like a camera. But instead of an exposure having been made the opposite had happened - an inclosure - a shutting down, a locking in. A moment before his head, his brain, were out in the world, seeing, hearing, sensing objects directly; now they were enclosed behind glass (like Crown jewels, like
Victorian wax fruit), behind a film - the film of the camera, perhaps, to continue the photographic analogy - a film behind which all things and people moved eerily, without colour, vivacity or meaning, grimly, puppet-like, without motive or conscious volition of their own..

A moment before his mind had heard and answered: now he was mentally deaf and dumb: he was in on himself - his mute, numbed self' (Hamilton 1941: p. 165).

doi: 10.1192/apt.20.5.365 